University of Nebraska - Lincoln

DigitalCommons@University of Nebraska - Lincoln

August 2005

\title{
Pyroelectric study of polarization switching in Langmuir-Blodgett films of poly(vinylidene fluoride trifluoroethylene)
}

\author{
A.V. Sorokin \\ University of Nebraska - Lincoln \\ V.M. Fridkin \\ University of Nebraska - Lincoln \\ Stephen Ducharme \\ University of Nebraska, sducharme1@unl.edu
}

Follow this and additional works at: https://digitalcommons.unl.edu/physicsducharme

Part of the Physics Commons

Sorokin, A.V.; Fridkin, V.M.; and Ducharme, Stephen, "Pyroelectric study of polarization switching in Langmuir-Blodgett films of poly(vinylidene fluoride trifluoroethylene)" (2005). Stephen Ducharme Publications. 3.

https://digitalcommons.unl.edu/physicsducharme/3

This Article is brought to you for free and open access by the Research Papers in Physics and Astronomy at DigitalCommons@University of Nebraska - Lincoln. It has been accepted for inclusion in Stephen Ducharme Publications by an authorized administrator of DigitalCommons@University of Nebraska - Lincoln. 


\title{
Pyroelectric study of polarization switching in Langmuir-Blodgett films of poly(vinylidene fluoride trifluoroethylene)
}

\author{
A. V. Sorokin, ${ }^{\text {a) }}$ V. M. Fridkin, ${ }^{\text {b) }}$ and Stephen Ducharme ${ }^{\text {c) }}$ \\ Department of Physics and Astronomy and Center for Material Research and Analysis, \\ University of Nebraska, Lincoln, Nebraska, 68588-0111
}

(Received 7 April 2005; accepted 27 June 2005; published online 24 August 2005)

\begin{abstract}
The ferroelectric switching in Langmuir-Blodgett films of poly(vinylidene fluoride trifluoroethylene) is studied. The films have a distribution of switching times several decades wide. Nearly a half of the film volume may be switched faster than $1 \mathrm{~ms}$, though complete switching of a whole sample may require $100 \mathrm{~s}$ or more. The switching occurs through a domain nucleation and growth mechanism. The decay of polarization at zero bias is logarithmic in time, with a constant rate below $5 \%$ per decade at room temperatures. The coercive voltage may be as low as $5 \mathrm{~V}$, which makes the films promising for use in nonvolatile random-access data storage. () 2005 American Institute of Physics. [DOI: 10.1063/1.2006228]
\end{abstract}

\section{INTRODUCTION}

Polyvinylidene fluoride (PVDF) and its copolymers are of great scientific and applied interest due to their pyro-, piezo-, and ferroelectric properties. ${ }^{1-3}$ Most often these materials are studied and used in a form of thin films, which may be prepared by solution casting, dipping, or spinning, as well as by more sophisticated techniques such as vacuum evaporation or electrospraying. In most thin-film works, films were down to several hundred nanometers thick. Spun films of a thickness down to $20 \mathrm{~nm}$ have been reported recently. ${ }^{4-7}$ In a technique developed in the mid-1990s, ${ }^{8-10}$ the Langmuir-Blodgett (LB) method is used to produce ultrathin ordered films of these ferroelectric polymers. A layer of polymer molecules is first formed on a water surface and then deposited onto a solid substrate. The surface layer on water can be, although not necessarily, just 1 molecule thick, and by its repeated transfer one can build a solid film with a predetermined thickness ranging from one nanometer to several microns. Such films of a PVDF and trifluoroethylene copolymer $\mathrm{P}(\mathrm{VDF} / \mathrm{TrFE})$ with a $70: 30$ composition have been prepared and intensively studied. ${ }^{11}$ Small thickness, low coercive voltage, low cost, and relatively easy fabrication make ferroelectric LB films promising for inexpensive nonvolatile random-access data storage devices. In very thin films the usual mechanism of polarization reversal through domain nucleation and growth may be suppressed, revealing the dynamics of intrinsic switching. ${ }^{12,13}$

The switching process in ferroelectrics may be studied by a variety of methods. The most often used are the measurement of charge or current hysteresis loops while a triangle or sine wave form is applied to a sample, and the measurement of current transients, arising in response to a voltage step. One limitation of these methods is a difficulty

\footnotetext{
a)Permanent address: Department of Physics, Ivanovo State University, Ivanovo 153025, Russia: a40in@mail.ru

${ }^{b)}$ Permanent address: Institute of Crystallography, Russian Academy of Sciences, Moscow 117333, Russia: fridkin@ns.crys.ras.ru

${ }^{c)}$ Electronic mail: sducharme1@unl.edu
}

to deal with a slow switching because of the dielectric relaxation time of the film. Also, these methods do not allow direct measurement of the steady polarization without disturbing it. The polarization state may be measured without significant disturbance, and the time range of studied switching processes may be extended by using the pyroelectric technique. It is based on the measurement of a voltage, current, or charge response to a heat pulse or wave due to a temperature dependence of a spontaneous polarization $P_{s}$ (primary pyroelectric effect). ${ }^{14}$ In a short-circuited thin-film capacitor the pyroelectric current density is

$$
j_{\mathrm{pyro}}=\eta \frac{d P_{s}}{d T} \frac{d T}{d t},
$$

where $d T / d t$ is the rate of change of temperature $T$ with respect to time $t$. The dimensionless factor $\eta$ takes into account the spatial distribution of the $\mathbf{P}_{s}$, and the component of $\mathbf{P}_{s}$ normal to the film. For instance, in an unpoled ferroelectric film $\eta=0$, because randomly oriented crystallites or domains cancel the response of each other, whereas in a material with fully aligned dipoles $\eta= \pm 1$. With a periodic temperature variation, modern lock-in amplifiers can detect the pyroelectric response caused by a very low excitation power, allowing nondestructive zero-field measurement of the steady polarization and the kinetics of very slow switching and relaxation processes in a short-circuited sample.

There are additional contributions to the current besides the direct pyroelectric effect described by Eq. (1). Another one, also proportional to the net polarization, arises from the secondary pyroelectric effect, which is the piezoelectric charge generated by the strain due to thermal expansion, and is influenced by constraints, such as the rigidity of the substrate. ${ }^{15,16}$ Because both primary and secondary pyroelectricity are proportional to $P_{s}$ and $\eta$, they need not be separated in the polarization reversal studies. In the PVDF family polymers with their complex morphology, a few other potential sources of current should be noted. This is, first, an inhomogeneously distributed charge, which may be injected from electrodes or otherwise trapped inside a polymer film. 
This charge will produce a displacement current during thermal expansion, but is independent of the film polarization. Depending on poling and sample storage conditions, the trapped charge may be metastable or unstable with a very different relaxation time, causing a temporal dependence of the measured response. Also, there may be some metastable polarization in the amorphous phase, or conversion of between amorphous and crystalline phases, both of which may contribute to the measured pyroelectric response and are dependent on sample history. ${ }^{2,17,18}$ The trapped charge and polarization currents from metastable dipole reorientation are not proportional to the spontaneous polarization and so care must be taken to distinguish contributions from these sources to the measured current. Studies of electron-irradiated copolymer LB films with amorphous fraction up to $100 \%$ have shown that the measured charge hysteresis is proportional to the crystalline fraction, and hence to the spontaneous polarization. $^{19,20}$

The pyroelectric technique is often used to study switching in ferroelectric polymer LB films, ${ }^{21-23}$ especially in the case of slow $(>1 \mathrm{~s})$ time scales when direct charge measurement is difficult. The pyroelectric response of the LB films of the 70:30 $\mathrm{P}(\mathrm{VDF} / \mathrm{TrFE})$ copolymer does not differ much from much thicker solvent-formed films. ${ }^{16}$ The stable polarization reversal was found even in thinnest films down to few transferred layers $(<10 \mathrm{~nm})$, showing no evidence of suppression of ferroelectricity by finite-size effects. ${ }^{22}$ The LB films also have relatively high coercive field $E_{c}$ of the order of $100 \mathrm{MV} / \mathrm{m}$, increasing with decreasing thickness. There is evidence of a saturation of $E_{c}$ to its intrinsic value for very thin films, attributed to the suppression of switching nucleation and domain growth. ${ }^{12}$ The switching in the thinnest films was found to be slow, in the range of seconds, ${ }^{13,16}$ in sharp contrast to the microsecond switching times typical with much thicker films formed by solvent spinning. ${ }^{24}$ Such a significant difference may be caused by suppression of nucleation or domain-wall motion. Switching times of $1-100 \mu$ s has been achieved with specially prepared LB films. ${ }^{25-27}$

To learn more about detailed polarization reversal and relaxation in LB films of the 70:30 $\mathrm{P}(\mathrm{VDF} / \mathrm{TrFE})$ copolymer, we measured the evolution of the pyroelectric response in short-circuited films after switching by voltage pulses with a duration ranging over nine decades, from $10 \mu \mathrm{s}$ to more than $10000 \mathrm{~s}$. We focused on the polarization evolution after the end of a voltage pulse, rather than on switching kinetics during the voltage application, as a function of the pulse height and duration, and the sample temperature. The response to multiple pulses, as well as the influence of the pulse duration on a pyroelectric hysteresis loop is investigated.

\section{EXPERIMENT}

Langmuir-Blodgett films $\mathrm{P}(\mathrm{VDF} / \mathrm{TrFE})$ 70:30 with intermediate thickness (20-35 transferred layers, 36-62 nm) were studied in the present work. These films are thick enough to be not influenced significantly by surface effects and inhomogeneities, but are still thin compared to spun films. The average film thickness of $1.78 \pm 0.07 \mathrm{~nm}$ per single

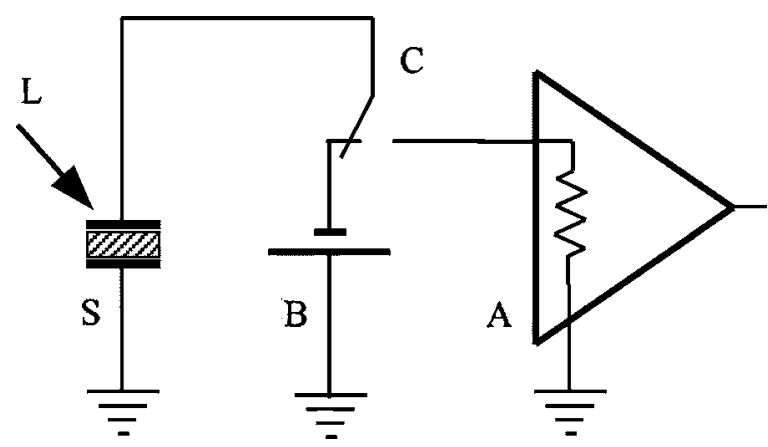

FIG. 1. Diagram of the pyroelectric measurement circuit. L, modulated laser beam; S, sample; B, voltage bias source; A, lock-in current amplifier; and C, relay switch.

deposited layer was determined by a spectroscopic ellipsometry method and confirmed by precise weighting and capacitance measurements. ${ }^{28}$

A Nima model 622 Langmuir trough filled with ultrapure $\left(>18 \mathrm{M} \Omega \mathrm{cm}\right.$ ) water, kept at $25{ }^{\circ} \mathrm{C}$ was used for film preparation. The surface layer on water was formed by drop-bydrop spreading of a $0.1 \%$ (by weight) solution of the copolymer in dimethyl sulfoxide with further compression by movable barriers starting from an area of $1200 \mathrm{~cm}^{2}$ at the rate of $60 \mathrm{~cm}^{2} / \mathrm{min}$. The films were deposited at a constant surface pressure of $5 \mathrm{mN} / \mathrm{m}$ on polished glass slides with evaporated Al stripes using the Schaefer (horizontal lift) variation of the Langmuir-Blodgett technique. After deposition, the samples were annealed at $120{ }^{\circ} \mathrm{C}$ for $24 \mathrm{~h}$. It is known that such annealing considerably improves the crystallinity and pyroelectric response of the films. ${ }^{29}$ Finally, top $\mathrm{Al}$ electrodes were evaporated to form up to sixteen $1 \times 1$ $\mathrm{mm}^{2}$ independent capacitors on each slide. The thickness of the top and bottom electrodes was approximately $100 \mathrm{~nm}$. The quality of the samples was checked by measuring their $\mathrm{dc}$ (with $1-\mathrm{V}$ test voltage) and ac $(50 \mathrm{mV}$ at $1 \mathrm{kHz})$ dielectric parameters. The test was passed if the capacitor's dc resistance was more than $20 \mathrm{M} \Omega$, capacitance was in the range of $40-60 \mathrm{nF} / \mathrm{mm}^{2}$ divided by the number of layers, and the dissipation factor $(\tan \delta)$ at $1 \mathrm{kHz}$ did not exceed 0.03 .

The Al electrodes usually deteriorate after prolonged voltage applications, due in part to weak spots in the copolymer film and underlying aluminum oxide layer, which break down under the high fields, causing some electrode material to oxidize. This leads to a decrease of the effective electrode area and to a reduction of the measured capacitance and pyroelectric response, which are proportional to the area. Each sample was trained at a moderate dc voltage (e.g., $12 \mathrm{~V}$ for 30-35 layer films) for 30-40 min. This removes or oxidizes the electrode over weak spots, where the film is thinner due to gaps in individual layers, so that they no longer contribute to electrical measurements.

For measurement of the pyroelectric current response, the sample was placed in a temperature-controlled chamber and connected to the circuit shown in Fig. 1. By convention, all potentials are measured at the top electrode with respect to the bottom one. Through the relay contacts, the sample is most of the time shorted by a low-impedance $(1 \mathrm{k} \Omega)$ current input of a Stanford Research Systems 830 lock-in amplifier, 
but could be quickly connected to a constant voltage source for pulse poling. Pulses ranging in duration from $1 \mu \mathrm{s}$ to $10 \mathrm{~s}$ were produced by an amplified Stanford Research Systems DG535 generator, longer pulses were created manually with a laboratory power supply. The pyroelectric response was measured by the Chynoweth optical modulation method, ${ }^{30}$ and is proportional to the net sample polarization. ${ }^{16}$ For thermal excitation of the film, the entire area of a capacitor under study was illuminated by a $5-\mathrm{mW}$ $\mathrm{He}-\mathrm{Ne}$ laser beam, chopped at a frequency of $2 \mathrm{kHz}$. Some fraction of the laser radiation was absorbed by the sample, producing a small modulation of its temperature at this frequency. Under these conditions, the temperature is uniform across the LB film thickness, ${ }^{31}$ and the sample temperature modulation amplitude is no more than $0.02{ }^{\circ} \mathrm{C}$. The lock-in amplifier time constant was $1 \mathrm{~s}$, and the recovery time after an overload, caused by the relay commutation, did not exceed $7 \mathrm{~s}$. The in-phase and orthogonal components of the lock-in output were recorded once per second by a computer and then used to calculate the pyroelectric current.

Experiments of two types were done.

(1) Poling and switching. The goal of this measurement is to find a relationship between the amount of switched polarization and amplitude and duration of a switching pulse. For each switching measurement, the sample was first poled by application of a fixed voltage large enough for a fixed time long enough to saturate the polarization nearly at its maximum value. The sample was then connected to the lock-in amplifier to measure the pyroelectric current, which was allowed to stabilize. After that, the sample was subjected to a pulse with opposite polarity, having a specific amplitude and duration. Finally, the sample was reconnected to the lock-in amplifier to measure the temporal dependence of the pyroelectric current. The resulting data consisted of pyroelectric current evolution curves recorded at different switching pulse amplitudes and durations, as well as at various sample temperatures.

(2) Hysteresis loops. The goal of this measurement was to determine the influence of the switching pulse amplitude and duration on the polarization hysteresis loop. The hysteresis loops were recorded by applying a sequence of relatively narrow pulses, according to a triangular envelope cycling between amplitude limits of opposite polarity. Each switching pulse was followed by a relatively long period at zero bias and a measurement of the pyroelectric response. The resulting data consisted of hysteresis loops of the pyroelectric current as a function of switching pulse amplitude and duration, and of the sample temperature.

Although the breakdown strength of the samples was high enough to allow 20-25-V pulses to be applied (for 30-layer samples, 375-470 MV/m), for quantitative comparison of the switched polarization it was necessary to prevent the degradation of electrodes. For this reason the amplitude was restricted to $16 \mathrm{~V}$ for pulses shorter than $100 \mathrm{~ms}$,

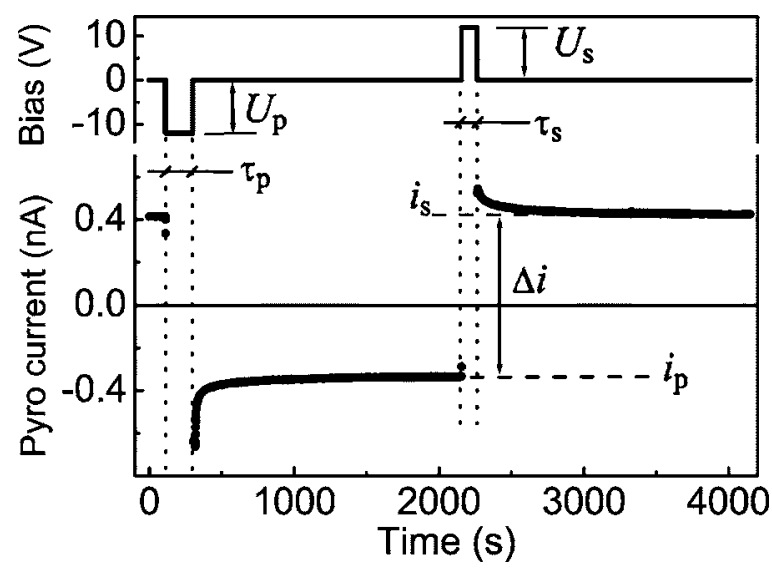

FIG. 2. A sequence of voltage pulses, used in poling-switching experiments, and an example of the pyroelectric current, recorded from a 35-layer film. The parameters $\tau_{p}, U_{p}, \tau_{s}$, and $U_{s}$ are durations and amplitudes of poling and switching pulses, respectively. The currents $i_{p}$ and $i_{s}$ are measured after relaxation, as shown. The net switched current is $\Delta i=i_{s}-i_{p}$.

and to $12 \mathrm{~V}$ for others. With these precautions, the samples were able to withstand hundreds of poling-switching cycles without noticeable damage.

\section{RESULTS}

\section{A. Poling and switching}

A typical voltage sequence and the corresponding pyroelectric response are shown in Fig. 2. A sample was first poled by a 200-s negative pulse with the amplitude well above a coercive voltage, which for a 35-layer film is approximately $7 \mathrm{~V}(112 \mathrm{MV} / \mathrm{m})$. After stabilization of the pyroelectric current, a positive switching pulse of variable amplitude and duration was applied to the sample. As can be seen from Fig. 2, a permanent change of the polarization due to ferroelectric switching is accompanied by a steady decrease of the pyroelectric response, which is especially pronounced immediately after the pulse. Although the decrease is not asymptotic, a few minutes after the end of the poling or switching pulse relaxation becomes very slow. These quasi-steady-state values of the pyroelectric current, $i_{p}$ and $i_{s}$, are a measure of the remanent polarization, and their difference $\Delta i=i_{s}-i_{p}$ is a measure of the switched polarization. In the experiment, we recorded the value of $i_{p} 30 \mathrm{~min}$ after the end of the poling pulse. For the value of $i_{s}$, this interval was varied from $5 \mathrm{~min}$ to several hours, because after shorter switching pulses the quasistable state is reached faster. The relaxation of polarization after switching, as well as the dependence of the switched polarization on the pulse duration, is shown in Fig. 3. Short pulses may not reverse the overall polarization sign, but do produce a new level of stable pyroelectric response, which persists well after the pulse, and reveals that permanent polarization reversal still occurs in a part of the sample.

The dependence of the switched pyroelectric current $\Delta i$ on the pulse amplitude and duration is shown in Fig. 4. Each point in the figure was obtained in a separate polingswitching cycle by measuring the $\Delta i$ value with the constant amplitude $U_{p}=-12 \mathrm{~V}(-193 \mathrm{MV} / \mathrm{m})$ and duration $\tau_{p}$ $=200 \mathrm{~s}$ of the poling pulse during the entire experiment. At 


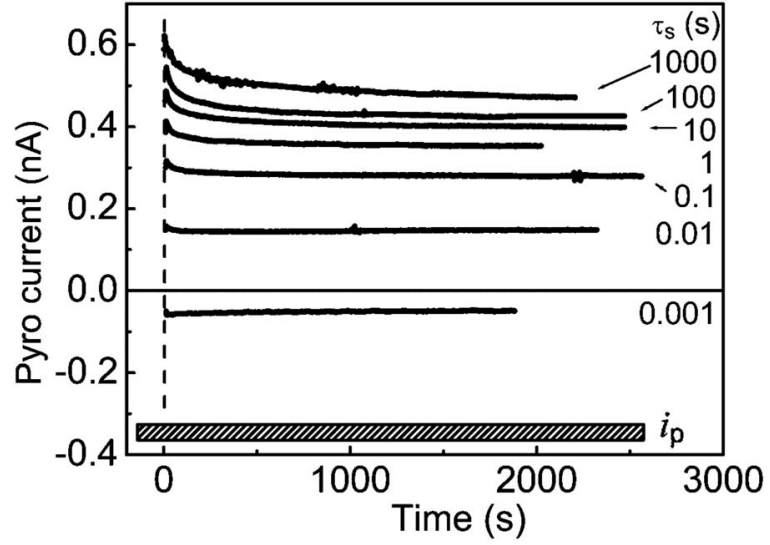

FIG. 3. The time evolution of the pyroelectric response in the 35-layer sample, recorded at room temperature after applying a switching pulse of amplitude $U_{s}=+12 \mathrm{~V}$ and duration $\tau_{s}$ indicated on the right. Zero time corresponds to the end of the switching pulse. The sample was poled with a pulse of amplitude $U_{p}=-12 \mathrm{~V}$ and duration $\tau_{p}=200 \mathrm{~s}$ and then short circuited for approximately $30 \mathrm{~min}$ before application of the switching pulses. The initial pyroelectric response $i_{p}$ for all the traces is within the hatched zone.

large amplitude, half of the sample's polarization may be switched by a 1-ms pulse, whereas a 1-s duration may be not enough to do so close to the apparent coercive voltage. To achieve full reversal of the polarization, and the maximum value of $\Delta i$, even the high amplitude pulse has to be sufficiently long, of the order of $100 \mathrm{~s}$. At the same time, even very low opposing voltage may produce measurable switching, if applied for a sufficiently long time, but will in general only partially switch the sample. There is a related trend in the dependence of $\Delta i$ on pulse amplitude-shorter pulses exhibit a relatively sharper voltage threshold.

The temperature dependence of the switched pyroelectric signal $\Delta i$ is mostly due to the strong dependence of the copolymer's pyroelectric coefficient on temperature. ${ }^{1,16}$ The pyroelectric current transients $\Delta i\left(\tau_{s}\right)$ in Fig. 5 show a steady increase in the pyroelectric signal as the temperature was increased from -17 to $+63{ }^{\circ} \mathrm{C}$, approaching the

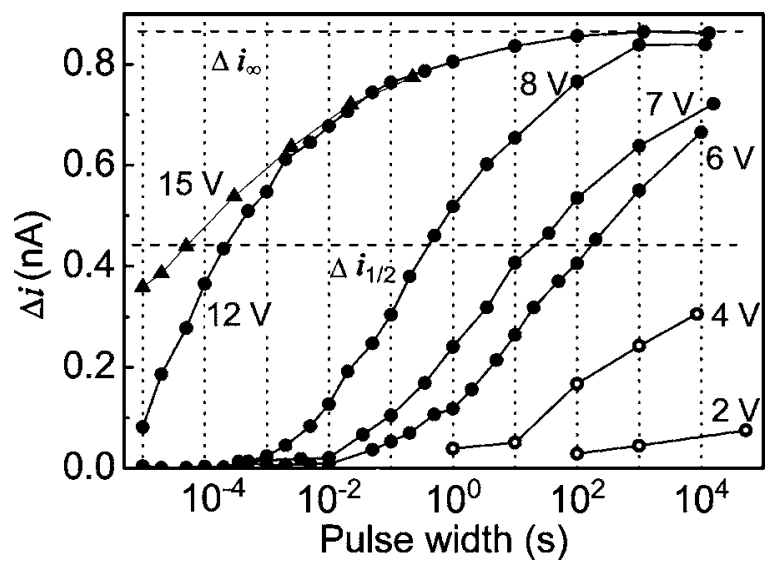

FIG. 4. The net change in pyroelectric signal $\Delta i=i_{s}-i_{p}$ due to switching with pulses of varying amplitude and duration recorded at room temperature with the 35-layer sample (solid symbols) and 30-layer sample (open circles). The poling pulse $\left(\tau_{p}=200 \mathrm{~s}, U_{p}=-12 \mathrm{~V}\right)$ was the same for all measurements.

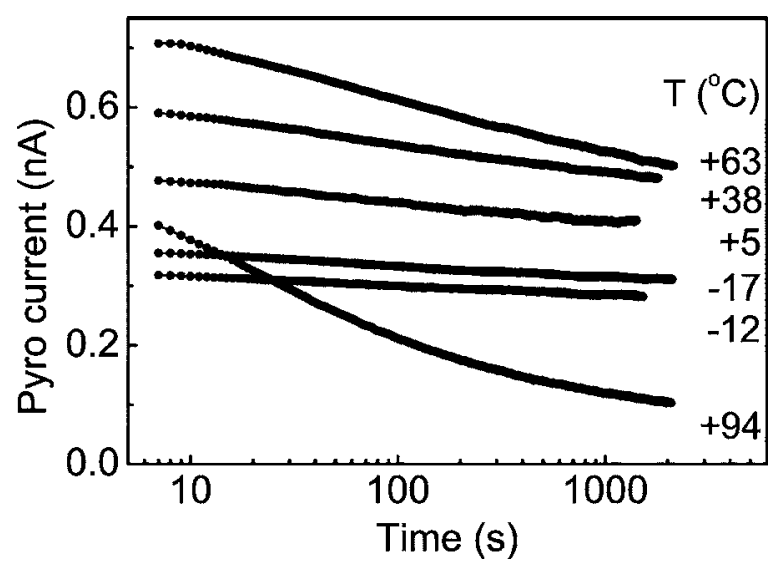

FIG. 5. The relaxation of pyroelectric response after the end of the switching pulse in the 30-layer sample as a function of sample temperature. The poling pulse $\left(\tau_{p}=200 \mathrm{~s}, U_{p}=-12 \mathrm{~V}\right)$ and switching pulse $\left(\tau_{p}=100 \mathrm{~s}, U_{p}=\right.$ $+12 \mathrm{~V}$ ) were the same for each measurement.

ferroelectric-paraelectric phase-transition temperature, and a precipitous drop at $94^{\circ} \mathrm{C}$, at the upper end of the phase coexistence region. ${ }^{1}$

An analysis of pyroelectric current transients can provide valuable information on polarization relaxation and retention. The relaxation of the pyroelectric response after a strong switching pulse of $+12 \mathrm{~V}(+193 \mathrm{MV} / \mathrm{m})$ applied for $100 \mathrm{~s}$ to a 30-layer film (Fig. 5) has a clear logarithmic dependence on time in a broad temperature range, except the region near the ferroelectric-to-paraelectric phase transition $\left(+94{ }^{\circ} \mathrm{C}\right.$ in Fig. 5). To compare the relaxation at different temperatures, it is convenient to use a relative decay rate, defined as

$$
\alpha=\frac{i(10 \mathrm{~s})-i(100 \mathrm{~s})}{i(10 \mathrm{~s})-i_{p}} 100 \%,
$$

where $i(t)$ is the pyroelectric current at a time moment $t$. (The time origin $t=0$ corresponds to the end of the switching pulse, but the lock-in recovery precludes the measurement of the first $7 \mathrm{~s}$ of the transient.) Because the pyroelectric signal is proportional to sample polarization, the parameter $\alpha$ also measures its decay rate, but is insensitive to the temperature dependence of the pyroelectric coefficient. The dependence of the decay rate $\alpha$ on the switching pulse duration and amplitude, and on sample temperature, is shown in Fig. 6. At temperatures below $40{ }^{\circ} \mathrm{C}$, where the $70: 30$ copolymer is fully ferroelectric, ${ }^{32}$ and for sufficiently large switching pulses, the decay rate does not exceed $5 \%$ per decade. This leads to $25 \%$ loss of the initial polarization in the first ten days, and $33 \%$ loss in the first three years, indicating good polarization retention. To verify this, we monitored the polarization decay in a short-circuited sample continuously for 7 days at room temperature, and measured a $22 \%$ loss over that period, in good agreement with the extrapolation based on the measurement of the decay rate during the first $30 \mathrm{~min}$ (Fig. 5).

As is evident in Figs. 3 and 4, switching by a single, relatively short pulse is incomplete. Further application of pulses of the same polarity, duration, and amplitude helps to switch more polarization, but after a certain number of 


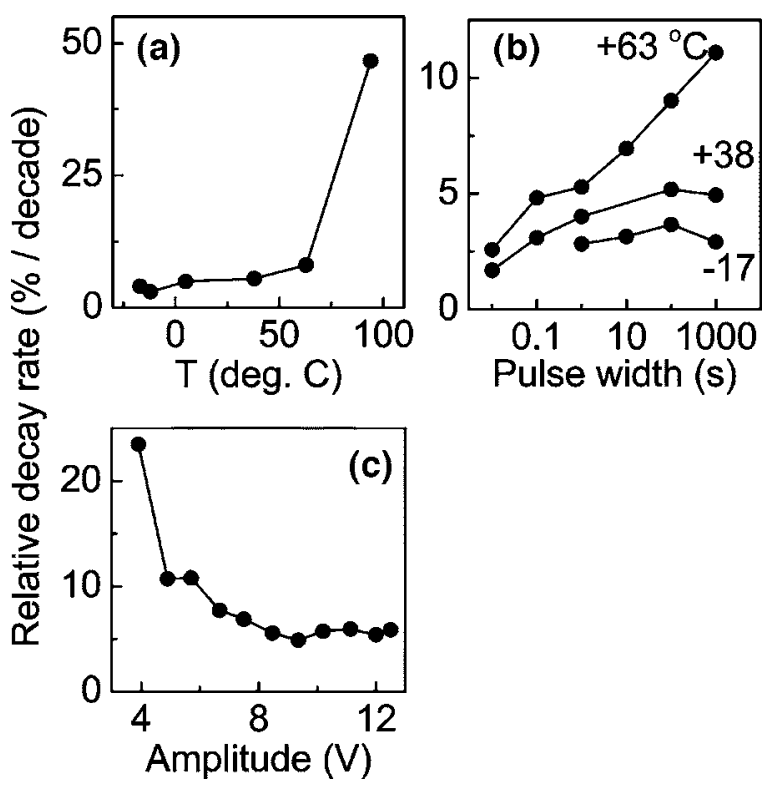

FIG. 6. The decadal pyroelectric relaxation rate $\alpha$ in the 30-layer film obtained from curves like those in Fig. 5. The poling pulse $\left(\tau_{p}=200 \mathrm{~s}, U_{p}\right.$ $=-12 \mathrm{~V}$ ) was the same for all measurements. (a) The dependence of the rate $\alpha$ on sample temperature for a switching pulse amplitude $U_{s}=+12 \mathrm{~V}$. (b) The dependence of the rate $\alpha$ on the switching pulse duration $\tau_{s}$ at different sample temperatures for a switching pulse amplitude $U_{s}=+12 \mathrm{~V}$. (c) The dependence of the rate $\alpha$ on the switching pulse amplitude $U_{s}$ for a sample temperature of $21^{\circ} \mathrm{C}$ and a switching pulse duration $\tau_{s}=100 \mathrm{~s}$.

pulses this effect reaches a maximum, but incomplete, polarization state, well below the value achievable in a long pulse of large amplitude (e.g., $12 \mathrm{~V}$ for $100 \mathrm{~s}$ as was used to obtain the relaxation data shown in Fig. 5). This accumulation effect is illustrated by Fig. 7(a), which shows the evolution of the pyroelectric signal while applying a series of positive $12-\mathrm{V}$ pulses of 1-ms duration to a negatively poled sample. The effect of pulse amplitude is illustrated in Fig. 7(b), which shows the quasistatic values of the pyroelectric current, measured after the $n$th pulse with a of duration $13 \mathrm{~ms}$, as a function of switching pulse amplitude $U_{s}$.

\section{B. Hysteresis loops}

To record a pyroelectric hysteresis loop, the 35-layer sample was subjected to square pulses with linearly changing amplitude, so that their envelope followed a triangle wave with a period of about $6 \mathrm{~h}$. The pulses with a duration from $1 \mathrm{~ms}$ to $100 \mathrm{~s}$ were separated by fixed pauses of $140 \mathrm{~s}$ at zero bias. The pyroelectric current was measured continuously and consisted of a series of transients, each followed by slow polarization variations with a period of the triangle wave envelope. Based on the above-described features of the polarization decay, the chosen duration of the pause may be considered insufficient to reach a stable state. Nevertheless, the value of the pyroelectric current recorded at the end of the pause may represent a very good approximation to the steady-state current. This is because the difference in the amplitudes of two consecutive pulses is just a fraction of volt, so the disturbance, caused by each new pulse, is much smaller than in the poling-switching experiments, and $140 \mathrm{~s}$ is enough time for the pyroelectric response to settle.

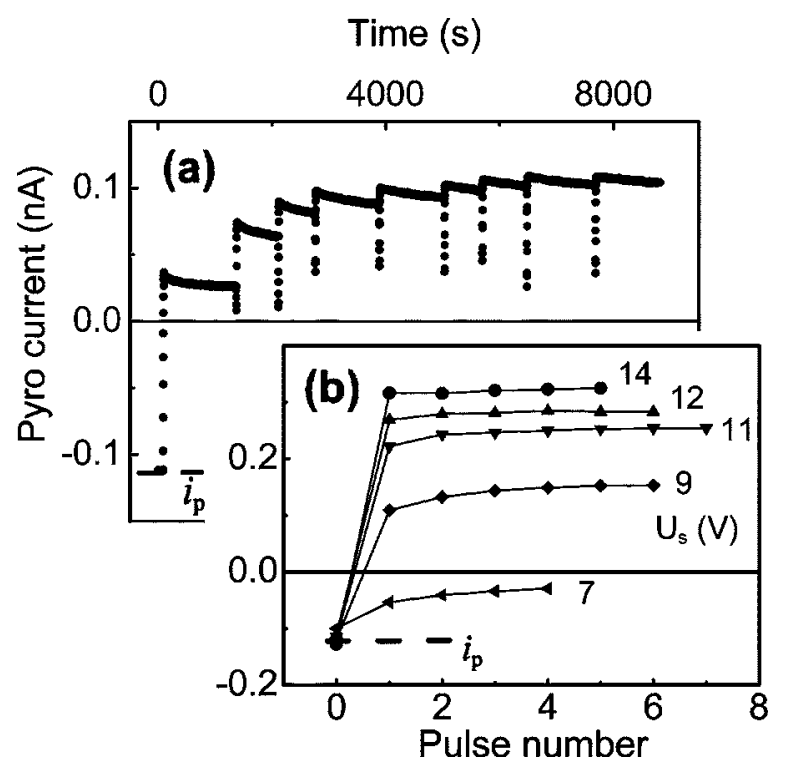

FIG. 7. Switching of a 30-layer film at room temperature by a series of switching pulses. A poling pulse $\left(\tau_{p}=200 \mathrm{~s}, U_{p}=-12 \mathrm{~V}\right)$ applied before each series. (a) Pyroelectric current measured during a series of pulses with amplitude $U_{s}=+12 \mathrm{~V}$ and duration $1 \mathrm{~ms}$. (b) Pyroelectric current measured immediately before each ensuing pulse in a series like the one shown in (a), but for a several different switching pulse amplitudes $U_{s}$.

The hysteresis loop cycles were repeated for several different pulse durations, starting at zero bias and zero polarization and cycling first toward negative bias. The raw pyroelectric data were processed to single out the value at $1 \mathrm{~s}$ before each new pulse, plotted versus the amplitude of the preceding pulse in Fig. 8(a). In order to erase the history of the sample and to start each loop from the virgin zeropolarization state, one or few pulses of varying polarity, duration, and amplitude were applied to get an approximately zero pyroelectric response. Such a depolarization of a ferroelectric film by a single pulse is a faster alternative to the use of multiple loop cycles with gradually decreasing amplitude. ${ }^{33}$ Figure $8(\mathrm{c})$ shows a typical processed experimental loop, including the initial segment from zero polarization at zero bias, recorded with a pulse duration of $10 \mathrm{~s}$.

Figure 8(a) shows three of the hysteresis loops, which were recorded with pulse durations of $1 \mathrm{~ms}, 0.1 \mathrm{~s}$, and $10 \mathrm{~s}$. The loop height is proportional to the switchable remanent polarization, and the loop width is a measure of the apparent coercive voltage. ${ }^{13}$ The dependence of the loop height and half-width on the pulse duration is shown in Fig. 8(b), showing that both scale approximately with the logarithm of the pulse duration. The maximum polarization reached in a loop is a result of the cumulative action of many pulses. Figure 8(c) compares the hysteresis loops obtained with small sequential pulses with steady polarization obtained with a single pair of $\pm 12.5-\mathrm{V}, 10$-s pulses. The accumulation of many 10 -s pulses results in only slightly larger polarization.

An interesting feature of the pyroelectric hysteresis loops is the presence of "bumps" that appear on decreasing pulse magnitude just after the amplitude extrema. These bumps are prominent in the 10-s loop in Figs. 8(a) and 8(c), and may be due to additional polarization similar to that observed with sequential pulses of the same amplitude (Fig. 

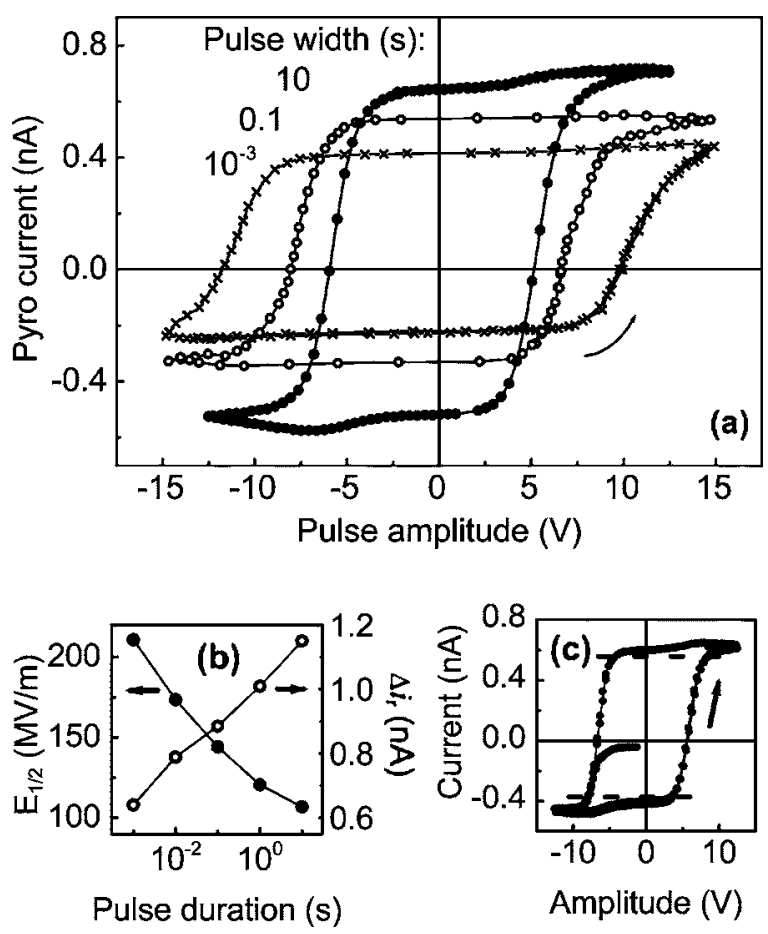

FIG. 8. Pyroelectric hysteresis measured at room temperature with a 35layer sample, obtained using a sequence of switching pulses with amplitudes cycling with a triangular envelope. (a) The hysteresis loops for three different pulse durations. (b) The dependence of the loop half-width $\left(E_{1 / 2}\right.$, left axis) and the remanent height $\left(\Delta i_{r}\right.$, right axis) on the pulse duration. (c) The full hysteresis loop for 10 -s pulses showing the initial segment starting a zero amplitude and zero sample polarization. The dashed lines show the values of the pyroelectric current measured after applying pulses of \pm 12.5 -V amplitude and 10-s duration, for comparison.

7). Both results are consistent with incomplete switching by a single pulse even by 10 -s pulses well above the coercive voltage. After the extrema the pulse amplitude is decreasing, but it is still high enough to switch the remaining parts of the film.

The results presented in this section were obtained with a few samples. It should be noted, though, that almost any sample that meets the minimal quality criteria shows similar switching characteristics. Sample-to-sample variation is noticeable, but typically the parameters, measured with different samples of equal thickness, vary by no more than $20 \%$, and the trends are the same. There is evidence of asymmetry in the hysteresis loops in Fig. 8 between the polarization states obtained with opposite polarities (of the top electrode relative to the bottom). The saturated pyroelectric current is larger after poling with a positive voltage, and a the negative coercive voltage is slightly larger. The asymmetry may be attributed to a fixed polarization at the border of the crystallites and amorphous phase, ${ }^{34}$ to a built-in electric field (though the electrodes are of the same material, they are not necessarily electrically symmetric), or to a polarization bias due to interactions with the substrate. These shifts are larger with thinner LB films, ${ }^{21,22,27}$ indicating the importance of surface interactions.

\section{DISCUSSION}

When ferroelectricity is concerned, the term switching is usually used to denote a change of the polarization sign by an external electric field. In our experiments an electric pulse permanently changes the magnitude of the polarization, and may reverse the sign if the pulse is of sufficient magnitude and duration. This means that the polarization may be reversed (switched) only in a part of the sample volume. Throughout this article we call switching any permanent change of the overall sample polarization, irrespective whether or not its sign was changed. Occasionally, we refer to switching data, obtained by other researchers with thicker melt-processed or solution-grown films. For brevity, we call all of them "spun films," having in mind the most-often used technique of solution spinning.

Summarizing the experimental results, one can point out the following features of the switching in ferroelectric polymer LB films.

(1) By applying pulses of different durations, amplitudes, and polarities, it is possible to set any stable value of sample polarization between the positive and negative maximum levels (Figs. 2 and 4). Some switching occurs even at very low pulse amplitude, but a large duration may be required to produce a significant polarization change (Figs. 3 and 4).

(2) The magnitude of the polarization change following a single pulse increases with the pulse amplitude and duration (Figs. 3 and 4). The effect of temperature is small except near the ferroelectric phase transition (Figs. 5 and 6).

(3) At given pulse amplitude and duration, the amount of switched polarization reaches a steady-state unsaturated value after a few pulses, but may be increased further by increasing either the amplitude or the duration of the pulses. Full saturation occurs at a large amplitude $(12 \mathrm{~V}$ for a 35-layer sample, or an electric field of $193 \mathrm{MV} / \mathrm{m}$ ) and very long duration of $100 \mathrm{~s}$ or more (Figs. 4 and 7). At the same time, a 1-ms pulse of sufficient amplitude may be enough to switch nearly half of the full saturated polarization (Fig. 4).

(4) When the switching pulse ends, the polarization slowly relaxes toward its initial value. The relaxation is logarithmic in time (Fig. 5), with the rate increasing with increased temperature and with increased switching pulse duration, and decreasing with increased pulse amplitude (Fig. 6). Well below the phase-transition temperature, the typical value of the decay rate is about $5 \%$ per a decade of time (Fig. 6).

(5) As the pulse duration increases, the width of the pyroelectric hysteresis loop (the apparent coercive field) decreases, while the height of the hysteresis loop (the switchable remanent polarization) increases, both logarithmically (Fig. 8).

These observations may be qualitatively explained by assuming that the films are inhomogeneous, and consist of many independent ferroelectric units, perhaps individual crystallite grains or domains, whose properties vary within some distribution. For example, each unit has its own critical pulse duration $\tau_{\mathrm{cr}}(E)$, a minimum duration of a pulse which at given amplitude may permanently reverse its polarization. A short positive pulse of duration $\tau_{s}$, applied to a negatively 
poled sample, will switch only a fraction of the units; the units with $\tau_{\mathrm{cr}}>\tau_{s}$ will remain in the original state. More and more units are involved as the pulse duration increases, thus increasing the amount of switched polarization (Fig. 4) and the height of the hysteresis loop. The loop's apparent coercive field reduces with increasing pulse duration [Fig. 8(b)] because longer pulses require smaller amplitude to switch an equal polarization. After the slowest unit switches, further increase of the pulse duration does not significantly increase the amount of the switched polarization, and it reaches a steady-state maximum value for that switching amplitude. Summarizing, the explanation is based on the two points: (i) the existence of a critical pulse duration $\tau_{\mathrm{cr}}(E)$ for each unit and (ii) the inhomogeneity of the film, which leads to a distribution of critical switching durations $\rho\left(\tau_{\mathrm{cr}}\right)$. In the following, the basic points are reviewed and the data from the Experiment section are discussed within the framework of this model.

The existence of the critical pulse duration can be drawn theoretically from a very basic model of domainless ("intrinsic") switching, described by the Landau-Khalatnikov equation. ${ }^{35}$ In the case of switching through domain nucleation and growth, there is also a minimum pulse duration that produces a stable domain with opposite polarization, because some time is required for a nucleus to appear and grow. The existence of the $\tau_{\mathrm{cr}}$ was shown experimentally, e.g., in triglycine sulfate (TGS), ${ }^{36}$ and the PVDF family polymers. ${ }^{2,37}$

At least two kinds of ferroelectric units, representing different levels of the inhomogeneity, may be identified in ferroelectric materials. These are, first, ferroelectric domains in single crystals, and, second, crystallites in polycrystalline materials, which may be in a single-domain state or subdivided into several domains. The units have different shapes, sizes, concentrations of defects, and other parameters. As a result, switching is highly inhomogeneous (or requires different pulse durations to initiate), even if a single crystal is concerned. In this case the volume ratio of domains with positive and negative polarizations gradually changes with the field on, and decays when the field is off, ${ }^{38,39}$ causing the temporal behavior of the overall polarization in some extent representative of the results reported here.

Spun films of PVDF and P(VDF/TrFE) consist primarily of lamellar or rod-shaped crystallites mixed with amorphous material. In as-prepared films, the crystallites may be as small as $10 \mathrm{~nm}$ along the chain direction, with considerably back-and-forth chain folding, whereas annealed films contain lamellar crystals of $0.1-0.2 \mu \mathrm{m}$ thick (along the chains) and 3-20 $\mu \mathrm{m}$ wide, ${ }^{40,41}$ depending on the film thickness and annealing conditions. ${ }^{42,43}$ The ferroelectric domain structure in the films is complex: a crystallite may consist of several domains, ${ }^{32,44}$ a domain may extend over many lamellar crystals stacked together, ${ }^{45}$ small crystallites may be in a single domain state. ${ }^{34,46}$ Because of a dependence of Curie temperature on the domain size and morphology, ${ }^{15}$ the ferroelectricto-paraelectric transition in spun films is smeared out over a wide temperature interval. ${ }^{1,47,48}$ Further, the defects leading to nucleation will also have their own distribution of activation energies. Therefore, the switching properties of indi-

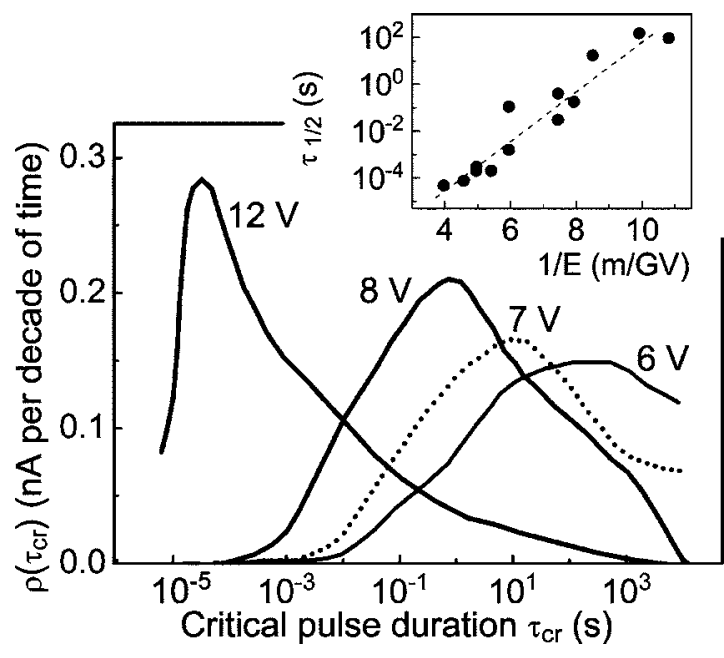

FIG. 9. The critical-switching-time distribution $\rho\left(\tau_{\mathrm{cr}}\right)=d(\Delta i) / d \tau_{s}$ obtained by differentiating and smoothing the experimental relaxation curves (Fig. 4). Inset: the pulse duration $\tau_{1 / 2}$ necessary to switch half of the polarization as a function of reciprocal electric field $E$.

vidual domains vary, causing the strong dependence of the reversed polarization on the pulse duration. $37,49,50$

The ferroelectric copolymer LB films show a similarly broad phase transition ${ }^{8}$ and duration dependence of the switched polarization, ${ }^{13}$ indicating the presence of ferroelectric units with varied properties. The units, though, may be not the same as in spun films, because of completely different crystallization conditions. When the LB films are produced, a droplet of the diluted solution of the copolymer added to a large volume of water, which is poor solvent for the polymer, but is infinitely miscible with dimethylsulfoxide (DMSO). As a result, the solution concentration at the surface may increase much faster compared to the solution spinning technique, but the amount of the material available for crystal growth is much smaller. This also means that the polymer is not strictly confined to the surface, as would a good fatty acid amphiphile such as stearic acid. The surface layer on water is highly constrained, and experiences further structure transformations during compression and transfer onto a substrate. Recrystallization may occur in a multilayer LB film during storage and definitely takes place during annealing. The annealed LB films are highly crystalline, ${ }^{29,51}$ but we do not have any data on the structure of the surface layer on water. Scanning electronic microscopy and atomic force microscopy imaging show good film uniformity and low roughness, though there are ridges and isolated nanocrystals that stick out from the films over about $5 \%$ of the area. $^{29,52,53}$

In the context of the independent ferroelectric units model, the dependence of switched polarization on pulse duration represented by $\Delta i\left(\tau_{s}\right)$ (Fig. 4) is proportional to the integral over the distribution $\rho\left(\tau_{\mathrm{cr}}\right)$ up to $\tau_{s}$. The derivative $d(\Delta i) / d \tau_{s}$, then, measures $\rho\left(\tau_{\mathrm{cr}}\right)$, the relative density of the units with critical switching time $\tau_{\mathrm{cr}}$. The distribution calculated in this way is shown in Fig. 9, and exhibits large dispersion revealed in the large distribution width of three to four decades in pulse duration, especially at amplitudes close to the nominal coercive voltage. As the pulse amplitude de- 
creases, the position of the maximum density shifts to longer pulse duration, with a rate of approximately -1 decade/V (with the 30-layer sample, $1 \mathrm{~V}$ corresponds to $19.6 \mathrm{MV} / \mathrm{m}$ ). The reason for the dispersion is not clear, but it has been noted in many studies of kinetics in films of VDF copolymers made by various methods. ${ }^{13,47,48,54-58}$ Some of the dispersion can be attributed to sample inhomogeneitymesoscopic structures such as grain boundaries and strain would affect local transition temperatures, coercive fields, and switching dynamics. Thickness and density variations would affect the electric-field distribution, broadening the apparent switching times. But these extrinsic causes of dispersion may not be the whole story. Studies of polarization kinetics near the phase-transition temperature show evidence of critical behavior that may arise from long-range polarization fluctuations. ${ }^{13,54,55}$ This may be connected with the large thermal hysteresis of the phase transition in the VDF copolymers. ${ }^{1,59-61}$ Further study of the nature and causes of kinetic dispersion is clearly necessary.

Different criteria are used in practice to characterize the switching speed. One useful criterion is the time of a switching current peak relative to the leading edge of the voltage step. Another is the time required to complete the reversal of $50 \%$ or $90 \%$ of the polarization. For the purpose of this work we used the parameter $\tau_{1 / 2}$, the duration of a pulse that reverses one-half of the total switchable polarization $\Delta i(\infty)$ (i.e., that leaves the poled sample with zero net polarization). Experimentally $\tau_{1 / 2}$ may be determined from the intersection of the curve $\Delta i\left(\tau_{s}\right)$ with a horizontal line $\Delta i_{1 / 2}=\Delta i(\infty) / 2$ (Fig. 4). The maximum value $\Delta i(\infty)$ is measured with a pulse of sufficient amplitude and duration to fully reverse and saturate the polarization (e.g., $\tau_{s}=1000 \mathrm{~s}$ and $U_{s}=12 \mathrm{~V}$ for films of 30-35 layers in thickness). From a statistical point of view, the value of $\tau_{1 / 2}$ is the median of the distribution of the ferroelectric units over $\tau_{\mathrm{cr}}$. The dependence of $\tau_{1 / 2}$ on the reciprocal electric field, shown in the inset to Fig. 9, coincides qualitatively with an exponential dependence of the switching time on field, $t_{\mathrm{sw}} \sim \exp \left(E_{a} / E\right)$, which is observed in many ferroelectrics, ${ }^{15,36,62,63}$ and can be explained in the domain nucleation and growth model. ${ }^{15,64,65}$ The results in Fig. 9 illustrate the noncritical nature of nucleated switching implied by the exponential dependence. With nucleation, there is no true coercive field, below which switching cannot occur, only an apparent threshold field that decreases with increased pulse duration. The lack of a true coercive field is a reflection of the activated nature of nucleation (the parameter $E_{a}$ has a term reciprocal in temperature ${ }^{62}$ ). Recently, films showing a true threshold coercive field were prepared and studied. ${ }^{13}$ Presumably, nucleation and domain growth are suppressed in these films, and they can be modeled with mean-field theory, ${ }^{35,66}$ leading to a square-root dependence of the form $\tau \sim\left(E-E_{C}\right)^{-1 / 2}$. ${ }^{13}$

The activation field $E_{a}$ is found to be $2.4 \mathrm{GV} / \mathrm{m}$ at about $300 \mathrm{~K}$. In spun films, $E_{a}=(0.75-0.9) \mathrm{GV} / \mathrm{m}$, as determined with the current peak method. ${ }^{1,67}$ It should be stressed that in a system with a distribution of $\tau_{\text {cr }}$ the overall value of the switching time $t_{\mathrm{sw}}$, measured with the current peak method, will not necessarily coincide with the $\tau_{1 / 2}$, unless the median of the distribution density coincides with its maximum. This is because the current is proportional to the distribution density, whereas the switched polarization is an integral of this function. For highly asymmetric distributions, like shown in Fig. 9, $t_{\mathrm{sw}}$ will always be smaller than $\tau_{1 / 2}$, and this is possibly the main reason for the observed discrepancy among different determinations of $E_{a}$.

Not all the polarization acquired by the end of the switching pulse is stable (Fig. 5). The decay rate is not high, and majority of the film retains its polarization for a very long time. The logarithmic decay, which does not seem to stop, may suggest that the polarization is thermodynamically unstable, but the validity of the logarithmic law for a very long time has not been checked carefully yet. It is possible that the polarization levels out at longer time scales. A sample stored for 14 months at room temperature and zero bias retained at least $75 \%$ of its pyroelectric response. The long-term stability of the polarization in LB films is similar to that found in spun films of PVDF-family polymers. ${ }^{68}$

There are several possible reasons for the observed decay of the pyroelectric response. If small crystallites are in a single-domain state and switch through an intrinsic mechanism, then those with $\tau_{\mathrm{cr}}>\tau_{s}$ will relax back to the original polarization state. The relaxation can be very slow, if the damping constant is high. ${ }^{13}$ In crystallites that switch through domain nucleation and growth, some of the nuclei grown during the field-on phase may become unstable when the field is turned off. The unstable contribution to the pyroelectric response may come also from the charge injected from electrodes and trapped at crystallite boundaries. ${ }^{18,37,49,69-71}$ Reversible electric-field conversion of paraelectric phase into ferroelectric may also contribute to the decay, especially prominent at elevated temperatures.

\section{CONCLUSIONS}

Polarization switching was studied in ferroelectric Langmuir-Blodgett films of poly(vinylidene fluoridetrifluoroethylene) by means of pyroelectric response measurements. It was shown that the switching kinetics are highly dispersive, indicating an inhomogeneous film. Half of the polarization can be reversed by a pulse shorter than $1 \mathrm{~ms}$, whereas for complete switching a 100-s pulse may be not enough. As a result, the apparent coercive field, determined from a pyroelectric hysteresis loop, decreases as the pulse duration is increased. The switching time increases exponentially with the reciprocal of the electric field; there is no threshold field, and the partial switching is possible even at very low voltage. This indicates that in the studied films the switching occurs through nucleation and domain growth mechanisms. After the switching pulse is concluded, the polarization relaxes toward the original state with a logarithmic dependence on time, with most of the relaxation occurring in the first few minutes, but there may be a leveling off of the polarization at about $75 \%$ of the initial value stable for a year or more.

For an explanation of the experimental data, the film was considered as an ensemble of independent ferroelectric units. 
Each unit has its own minimum pulse duration necessary for switching at a given switching field. Basing on this assumption, it was shown that the distribution of the units over critical pulse durations is asymmetric and very broad, covering several decades on time scale. To improve switching characteristics, and possibly reduce relaxation, one should suppress long tale of the slowest part of the distribution, which would require a deeper understanding of the origins of the phenomenon. By solving this problem, one can hold a hope that the films with their low coercive voltage and good polarization retention may be useful for application in inexpensive portable information, computing, and smart card applications, where data persistence, low cost, and low power are at a premium.

\section{ACKNOWLEDGMENTS}

This work was supported by the National Science Foundation, the Office of Naval Research, and the Nebraska Research Initiative.

${ }^{1}$ T. Furukawa, Phase Transitions 18, 143 (1989).

${ }^{2}$ G. Eberle, H. Schmidt, and W. Eisenmenger, IEEE Trans. Dielectr. Electr. Insul. 3, 624 (1996).

${ }^{3}$ K. Tashiro, in Ferroelectric Polymers, edited by H. S. Nalwa (Marcel Dekker, New York, 1995), pp. 63-181.

${ }^{4}$ A. Guy and I. Guy, Integr. Ferroelectr. 14, 259 (1997).

${ }^{5}$ A. Limbong and I. L. Guy, Thin Solid Films 325, 187 (1998).

${ }^{6}$ A. Limbong, I. Guy, Z. Zheng, Afifuddin, and T. Tansley, Ferroelectrics 230, 61 (1999).

${ }^{7}$ X. Q. Chen, H. Yamada, Y. Terai, T. Horiuchi, K. Matsushige, and P. S. Weiss, Thin Solid Films 353, 259 (1999).

${ }^{8}$ S. Palto, L. Blinov, A. Bune, E. Dubovik, V. Fridkin, N. Petukhova, K. Verkhovskaya, and S. Yudin, Ferroelectr., Lett. Sect. 19, 65 (1995).

${ }^{9}$ L. M. Blinov, V. M. Fridkin, S. P. Palto, A. V. Sorokin, and S. G. Yudin, Thin Solid Films 284-85, 469 (1996).

${ }^{10}$ A. Sorokin, S. Palto, L. Blinov, V. Fridkin, and S. Yudin, Mol. Mater. 6, 61 (1996).

${ }^{11}$ S. Ducharme, S. P. Palto, and V. M. Fridkin, in Ferroelectric and Dielectric Thin Films, edited by H. S. Nalwa (Academic, San Diego, 2002), Vol 3.

${ }^{12}$ S. Ducharme, V. M. Fridkin, A. V. Bune, S. P. Palto, L. M. Blinov, N. N. Petukhova, and S. G. Yudin, Phys. Rev. Lett. 84, 175 (2000).

${ }^{13}$ G. Vizdrik, S. Ducharme, V. M. Fridkin, and S. G. Yudin, Phys. Rev. B 68, 094113 (2003)

${ }^{14}$ S. Bauer and S. B. Lang, IEEE Trans. Dielectr. Electr. Insul. 3, 647 (1996).

${ }^{15}$ M. E. Lines and A. M. Glass, Principles and Applications of Ferroelectrics and Related Materials (Clarendon, Oxford, 1977).

${ }^{16}$ A. V. Bune, C. Zhu, S. Ducharme, L. M. Blinov, V. M. Fridkin, S. P. Palto, N. N. Petukhova, and S. G. Yudin, J. Appl. Phys. 85, 7869 (1999).

${ }^{17}$ D. Rollik, S. Bauer, and R. Gerhard-Multhaupt, J. Appl. Phys. 85, 3282 (1999).

${ }^{18}$ H. von Seggern and S. Fedosov, IEEE Trans. Dielectr. Electr. Insul. 11, 232 (2004).

${ }^{19}$ C. M. Othon and S. Ducharme, Ferroelectrics 304, 9 (2004).

${ }^{20}$ C. M. Othon, F. B. Bateman, and S. Ducharme, J. Appl. Phys. 98, 014106 (2005).

${ }^{21}$ L. M. Blinov, V. M. Fridkin, S. P. Palto, A. V. Sorokin, and S. G. Yudin Thin Solid Films 284-85, 474 (1996).

${ }^{22}$ A. V. Bune, V. M. Fridkin, S. Ducharme, L. M. Blinov, S. P. Palto, A. V. Sorokin, S. G. Yudin, and A. Zlatkin, Nature (London) 391, 874 (1998).

${ }^{23}$ V. V. Kochervinskii, B. V. Lokshin, S. P. Palto, G. N. Andreev, L. M. Blinov, and N. N. Petukhova, Polym. Sci., Ser. A Ser. B 41, 832 (1999).

${ }^{24}$ T. Furukawa, H. Matsuzaki, M. Shiina, and Y. Tajitsu, Jpn. J. Appl. Phys., Part 2 24, L661 (1985).

${ }^{25}$ S. Ducharme, S. P. Palto, L. M. Blinov, and V. M. Fridkin, in Fundamental Physics of Ferroelectrics, edited by R. E. Cohen, AIP Conference Proceedings (American Institute of Physics, Melville, New York, 2000), Vol. 535 , p. 354
${ }^{26}$ L. M. Blinov, V. M. Fridkin, S. P. Palto, A. V. Bune, P. A. Dowben, and S. Ducharme, Phys. Usp. 43, 243 (2000).

${ }^{27}$ A. R. Geivandov, S. P. Palto, S. G. Yudin, V. M. Fridkin, L. M. Blinov, and S. Ducharme, in Advanced Organic and Inorganic Optical Materials, 19-22 August 2002, edited by A. Krumins D. Millers, I. Muzikante, A. Sternbergs, and V. Zauls, Proceedings of the SPIE (SPIE-The International Society for Optical Engineering, Bellingham, WA, 2003), Vol. 5122, pp. 216-223.

${ }^{28}$ M. Bai, M. Poulsen, A. V. Sorokin, S. Ducharme, C. M. Herzinger, and V. M. Fridkin, J. Appl. Phys. 95, 3372 (2004).

${ }^{29}$ M. Bai, M. Poulsen, A. V. Sorokin, S. Ducharme, and V. M. Fridkin, in Electroactive Polymers and Their Applications as Actuators, Sensors, and Artificial Muscles, 26-30 November 2001, edited by Q. Zhang, E. Fukada, Y. Bar-Cohen, and S. Bauer, Proceedings of the MRS (Materials Research Society, Boston, 2002), Vol. 698, EE2.8.2-7.

${ }^{30}$ A. G. Chynoweth, J. Appl. Phys. 27, 78 (1956).

${ }^{31}$ B. Ploss, R. Emmerich, and S. Bauer, J. Appl. Phys. 72, 5363 (1992).

${ }^{32}$ J. F. Legrand, Ferroelectrics 91, 303 (1989).

${ }^{33}$ M. J. Higgins, A. Krishnan, M. M. J. Treacy, and S. Bhattacharaya, Appl. Phys. Lett. 80, 3373 (2002).

${ }^{34}$ B. Ploss and B. Ploss, IEEE Trans. Dielectr. Electr. Insul. 5, 91 (1998).

${ }^{35}$ L. D. Landau and I. M. Khalatnikov, Dokl. Akad. Nauk SSSR 96, 469 (1954).

${ }^{36}$ E. Fatuzzo and W. J. Merz, Phys. Rev. 116, 61 (1959).

${ }^{37}$ M. Womes, E. Bihler, and W. Eisenmenger, IEEE Trans. Dielectr. Electr. Insul. 24, 461 (1989).

${ }^{38}$ N. A. Romanyuk and I. S. Zheludev, Kristallografiya 5, 403 (1960).

${ }^{39}$ H. H. Wieder, J. Appl. Phys. 27, 413 (1956).

${ }^{40}$ K. Koga and H. Ohigashi, J. Appl. Phys. 59, 2142 (1986).

${ }^{41}$ H. Ohigashi, N. Kagami, and G. R. Li, J. Appl. Phys. 71, 506 (1992).

${ }^{42}$ K. Kimura and H. Ohigashi, Jpn. J. Appl. Phys., Part 1 25, 383 (1986).

${ }^{43}$ T. Fukuma, K. Kobayashi, T. Horiuchi, P. Yamada, and K. Matsushige, Jpn. J. Appl. Phys., Part 1 39, 3830 (2000).

${ }^{44}$ A. Wicker, B. Berge, J. Lajzerowicz, and J. F. Legrand, J. Appl. Phys. 66, 342 (1989).

${ }^{45}$ G. R. Li and H. Ohigashi, Jpn. J. Appl. Phys., Part 1 31, 2495 (1992).

${ }^{46}$ K. Tashiro, R. Tanaka, K. Ushitora, and M. Kobayashi, Ferroelectrics 171, 145 (1995).

${ }^{47}$ Y. Takahashi and T. Furukawa, Jpn. J. Appl. Phys., Part 1 39, 3511 (2000).

${ }^{48}$ A. Toda, Y. Takahashi, T. Arita, M. Hikosaka, and T. Furukawa, J. Chem. Phys. 114, 6896 (2001).

${ }^{49}$ G. Eberle, E. Bihler, and W. Eisenmenger, IEEE Trans. Dielectr. Electr. Insul. 26, 69 (1991).

${ }^{50}$ A. K. Tagantsev, I. Stolichnov, N. Setter, J. S. Cross, and M. Tsukada, Phys. Rev. B 66, 214109 (2002).

${ }^{51}$ J. Choi et al., Phys. Rev. B 61, 5760 (2000).

${ }^{52}$ S. Ducharme, M. Bai, M. Poulsen, S. Adenwalla, S. P. Palto, L. M. Blinov, and V. M. Fridkin, Ferroelectrics 252, 191 (2001).

${ }^{53}$ M. Bai, Ph.D. thesis, University of Nebraska, 2002

${ }^{54}$ T. Furukawa and G. E. Johnson, J. Appl. Phys. 52, 940 (1981).

${ }^{55}$ R. L. Moreira, R. P. S. M. Lobo, G. Medeiros-Ribeiro, and W. N. Rodrigues, J. Polym. Sci., Polym. Phys. Ed. 32, 953 (1984).

${ }^{56}$ J. R. Gregorio and E. M. Ueno, J. Mater. Sci. 34, 4480 (1999).

${ }^{57}$ S. P. Palto, A. M. Lotonov, K. A. Verkhovskaya, G. N. Andreev, and N. D. Gavrilova, JETP 90, 301 (2000).

${ }^{58}$ A. R. Geivandov, S. P. Palto, S. G. Yudin, and L. M. Blinov, JETP 99, 83 (2004).

${ }^{59}$ K. Tashiro, M. Nakamura, M. Kobayashi, Y. Chatani, and H. Tadokoro, Macromolecules 17, 1452 (1984).

${ }^{60}$ K. Koga, N. Nakano, T. Hattori, and H. Ohigashi, J. Appl. Phys. 67, 965 (1990).

${ }^{61}$ E. Lopez Cabarcos, A. F. Brana, B. Frick, and F. Batallan, Phys. Rev. B 65, 104110 (2002).

${ }^{62}$ W. J. Merz, Phys. Rev. 95, 690 (1954).

${ }^{63}$ W. J. Merz, J. Appl. Phys. 27, 938 (1956).

${ }^{64}$ E. Fatuzzo, Phys. Rev. 127, 1999 (1962).

${ }^{65}$ Y. Ishibashi and Y. Takagi, J. Phys. Soc. Jpn. 31, 506 (1971).

${ }^{66}$ D. Ricinschi, C. Harnagea, C. Papusoi, L. Mitoseriu, V. Tura, and M. 
Okuyama, J. Phys.: Condens. Matter 10, 477 (1998).

${ }^{67}$ A. Konno, K. Shiga, H. Suzuki, T. Koda, and S. Ikeda, Jpn. J. Appl. Phys., Part 1 39, 5676 (2000).

${ }^{68}$ A. G. Kolbeck, J. Polym. Sci., Polym. Phys. Ed. 20, 1987 (1982).

${ }^{69} \mathrm{~W}$. Eisenmenger, in 9th International Symposium on Electrets Proceed- ings, edited by X. Zhongfu and Z. Hongyan (IEEE, New York, 1996), p. 813 .

${ }^{70}$ G. F. Leal Ferreira and R. A. Moreno, J. Appl. Phys. 75, 472 (1994).

${ }^{71}$ H. von Seggern and S. Fedosov, IEEE Trans. Dielectr. Electr. Insul. 7, 543 (2000). 